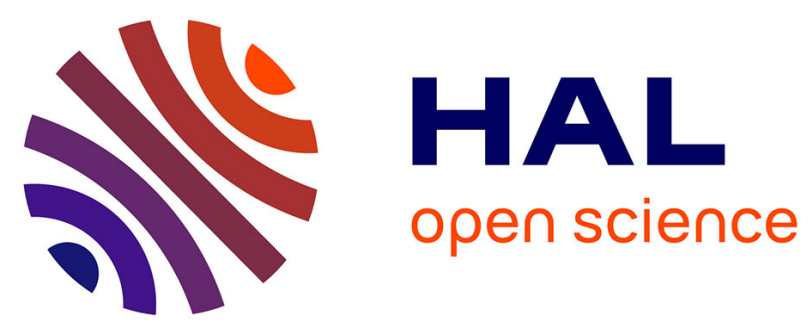

\title{
Dédale et Icare du XIIe au XVe siècle : artifice et arts mécaniques au Moyen Age
}

Christine Ferlampin-Acher

\section{To cite this version:}

Christine Ferlampin-Acher. Dédale et Icare du XIIe au XVe siècle: artifice et arts mécaniques au Moyen Age. T. Picard et E. Lavezzi. L'artifice dans les lettres et les arts, Presses universitaires de Rennes, p. 273-284, 2015, 978-2-7535-4145-0. hal-01627673

\section{HAL Id: hal-01627673 https://hal.science/hal-01627673}

Submitted on 2 Nov 2017

HAL is a multi-disciplinary open access archive for the deposit and dissemination of scientific research documents, whether they are published or not. The documents may come from teaching and research institutions in France or abroad, or from public or private research centers.
L'archive ouverte pluridisciplinaire HAL, est destinée au dépôt et à la diffusion de documents scientifiques de niveau recherche, publiés ou non, émanant des établissements d'enseignement et de recherche français ou étrangers, des laboratoires publics ou privés.

\section{(1)(1) $\$(0)$}

Distributed under a Creative Commons Attribution - NonCommercial - ShareAlikel 4.0 
Dédale et Icare du XIIe au XVe siècle : artifice et arts mécaniques au Moyen Age Christine Ferlampin-Acher

«Dédale et Icare du XIIe au XVe siècle : artifice et arts mécaniques au Moyen Âge », dans L'artifice dans les lettres et les arts, T. Picard et E. Lavezzi (dir.), Rennes, Presses universitaires de Rennes, 2015, p. 273-284 ;

Le point de départ de cette réflexion est un travail réalisé dans le cadre du séminaire des médiévistes de Rennes 2, dans lequel je m'interrogeai sur la présence de Dédale dans la traduction de l'Historia Regum Britaniae qui figure dans le vaste roman en prose de Perceforest, conservé dans des manuscrits du XVe siècle ${ }^{1}$. La fabrication des ailes artificielles par Dédale et le vol fatal d'Icare, dans la chronique latine en vers de Geoffroy de Monmouth (entre 1135 et 1138) sert de patron à la figure de Bladud, ce que reprend Perceforest à son tour à la fin du Moyen Age lorsqu'il donne une traduction de l'œuvre latine en amont de son texte : David Aubert, à qui l'on doit la version de Perceforest datée de 14459-1460 conservée à la Bibliothèque de l'Arsenal de Paris, introduit une modification qui tire explicitement l'évocation vers le modèle de la charpenterie de marine ${ }^{2}$.

Les ailes artificielles de Dédale et Icare soulèvent le problème de l'artifice fabriqué par les humains et imitant la nature. Cependant si la thématique antique de l'homme victime de son hybris pouvait être relue dans une perspective chrétienne condamnant, dans la même perspective augustinienne que celle qui condamne la métamorphose, celui qui veut se substituer au divin Créateur, Dédale et Icare semblent susciter au Moyen Age des lectures diverses : un parcours, nécessairement limité dans le cadre d'un article, attestera d'une part du succès de Dédale et Icare tout au long du Moyen Age et d'autre part de l'éclatement du mythe et de sa valorisation diverse : l'artifice des ailes peuvent être valorisé positivement.

Cependant l'exemple de Perceforest, en particulier dans la version de David Aubert, montre que les ailes artificielles de Dédale sont imaginées sur le modèle de l'ouvrage du charpentier de marine, ce qui renvoie aux arts mécaniques, opposés aux arts libéraux ${ }^{3}$. Les arts mécaniques, depuis leur mention dans le commentaire de Scott Erigène au IXe siècles des

\footnotetext{
${ }^{1}$ Voir mon article «Les ailes volantes dans Perceforest: Dédale, Icare, Phaéton et Elie », à paraître dans le volume Machine et machinerie au Moyen Age, sous la direction de F. Pomel, Rennes, Presses Universitaires de Rennes.

${ }^{2}$ L'épisode de Perceforest se lit dans Perceforest. Première partie, éd. Gilles Roussineau, Genève, Droz, Textes littéraires français, 2 t., 2007, t. I, p 36-37. Sur cette traduction, voir G. Veysseyre, "L'Historia regumeBritannie, ou l'enfance de Perceforest », dans Enfances arthuriennes, éd. D. Hüe et C. Ferlampin-Acher, Rennes, Presses Universitaires de Rennes, 2006, p. 99-126.

${ }^{3}$ Pour une synthèse sur les arts mécaniques, voir Les arts mécaniques au Moyen Age,Cahiers d'études médiévales, éd. G. H. Allard et S. Lusignan, t. 7, Montréal, Bellarmin, et Paris, J. Vrin, 1982, en particulier la première partie, due à G. Allard, qui ressitue les arts mécaniques dans la perspective de l'idéologie médiévale.
} 
Noces de Philologie et Mercure de Martianus Capella ${ }^{4}$ et l'énumération qu'en donne Hugues de Saint-Victor vers 1125 dans son Didascalicon ${ }^{5}$ sont dénombrés par opposition aux sept arts libéraux qui constituent la base de l'enseignement antique puis médiéval. Les sept arts mécaniques, définis comme imitation de la nature, sont qualifiés d' «adultérins » par Hugues de Saint-Victor car, contrairement aux arts libéraux, ils n'exigent pas un esprit libre. Cependant la dévaluation des arts mécaniques n'est pas systématique au Moyen Age: le travail manuel, pour Roger Bacon ou Raymond Lulle, peut être valorisé car il contribue à la perfection de l'être. Par ailleurs, l'opposition entre art et artisanat, peu pertinente au début du Moyen Âge, tend à s'affirmer progressivement, contribuant à une nouvelle organisation des pratiques, quand l'artifice devient artistique. Dans cette perspective, la constatation sur laquelle je concluais mon article sur Dédale et Icare dans Perceforest, à savoir que contrairement à d'autres figures antiques Dédale et Icare étaient évacués du reste du roman en prose, peut être éclairée d'un jour nouveau, si elle est envisagée dans la perspectives de l'artifice et de ses rapports avec les arts mécaniques. L'artifice qui fait de l'homme un oiseau (ou un poisson) et celui qui permet d'imiter le réel par l'illusion artistique ne sont pas traités de la même façon en cet «automne du Moyen Âge " ${ }^{6}:$ les ailes d'Icare, artisanales et utilitaires, s'effacent devant celles des déesses qui volent sur les scènes théâtrales.

I. Un mythe éclaté

Sans qu'il soit possible de prétendre à l'exhaustivité, quelques exemples attesteront du succès de Dédale et Icare tout au long du Moyen Age, et de la diversité des lectures qui sont faites de ces figures.

Plus présent qu'Icare, Dédale représente souvent le génie $\operatorname{architectural}^{7}$, la démesure de l'homo faber, mais aussi l'homme souffrant, le prisonnier -dans le labyrinthe-, comme chez Charles d'Orléans où il incarne la mélancolie typique de cette fin de Moyen Age:

C'est la prison Dedalus,

Que de ma merencolie,

Quant je la cuide faillie,

J'y rentre de plus en plus.

\footnotetext{
${ }^{4}$ S. Grebe, Martianus Capella, De Nuptiis Philologiae et Mercurii. Darstellung der Sieben Freien Künste und ihrer Beziehungen zueinander, Stuttgart-Leipzig, Teubner, 1999 et M. Bovey, Disciplinae cyclicae: L'organisation du savoir dans l'ouvre de Martianus Capella, Trieste, Edizioni Università di Trieste, 2003.

${ }_{6}^{5}$ Didascalion, II, 20-27 (Patrologie Latine, t. 176, col. 760-763).

${ }^{6}$ G. Roussineau date Perceforest du XIVe siècle. Je penche pour une datation au XVe : voir mon ouvrage Perceforest et Zéphir : propositions autour d'un récit arthurien bourguignon, Genève, Droz, 2010. L'expression «automne du Moyen Age » est empruntée à J. Huizinga (le titre de son ouvrage, paru en 1919, a d'abord été traduit par «La décadence du Moyen Age », puis «L'automne du Moyen Age », L'Automne du Moyen Age, Paris, Payot, coll. "Petite Bibliothèque Payot", 2002)
} 
Aucunes foiz je conclus

D'y bouter Plaisance lie ${ }^{8}$.

Cette diversité des lectures signale un éclatement de la figure : l'artiste et l'ingénieur qui se confondaient dans la figure antique, lorsque le même Dédale fabriquait des ailes volantes et décorait le temple qu'il avait fondé en Sicile, sont désormais désolidarisés ; si Dédale peut incarner le peintre poète, il est aussi, dans d'autres contextes, ingénieur.

D'un côté l'opprobre contre le vol audacieux, que met en scène Rigaud de Barbezieux, qui insiste sur la démesure sacrilège du vol, et que retiendront les recueils d'emblèmes du XVIe siècles $^{9}$ :

Ben sai qu'Amors es tan granz

Que leu me pot pardonar

S'eu falhi per sobramar

Ni renhei com Ycarus

Que dis qu'il era Jhesus

E vole volar al cel outracuidanz $?^{10}$

De l'autre les Pères de l'Eglise ${ }^{11}$ ou l'Ovide Moralisé qui donnent du vol d'Icare et Dédale une lecture chrétienne valorisante et y voient une image de l'âme ${ }^{12}$.

\footnotetext{
${ }^{7}$ Op. cit., p. 30.

${ }^{8}$ Charles d'Orléans, Poésies, éd. P. Champion, éd. Honoré Champion, rééd. 2010, rondeau CCCCXI, v. 1. Voir Cl. Galderisi, « De 'La prison Dedalus' à la mélancolie de la captivité anglaise. Charles d'Orléans en la prison de poésie » dans Le loro prigioni. Scritture dal carcere, éd. Anna Maria Babbi et Tobia Zanon, Vérone, Fiorini, 2007, p. 97-115. On retrouve dans la Ballade contre les ennemis de la France de Villon, qui s'en prend aux Bourguignons, une allusion à la prison en la tour Dedalus (éd. Cl. Thiry, Paris, Lettres Gothiques, 1991, p. 271). L'allusion est vague, le labyrinthe semblant être confondu avec la tour d'où s'élancent le père et le fils pour prendre les airs. Jason et Dédale sont présents en contexte, ce qui est logique puisqu'il est question des Bourguignons, dont Jason est l'une des figures emblématiques. Le rapprochement entre Jason et Dédale me semble provoquer une confusion entre Icare et Jason lorsque dans la Ballade de Fortune (p. 305, v. 22) on lit: En mer noyay Jazon en ung boullon.

${ }^{9}$ M. Dancourt, op. cit., p. 41.

${ }^{10}$ Les chansons du troubadour Rigaud de Barbezieux, éd. J. Anglade, Montpellier, 1919, p. 61. La tradition dénonçant la presompcion du vol d'Icare, dont les ailes fondent au soleil, est vivace: Chrétien de Pizan l'exploite quand elle évoque sa montée aux cieux dans le Chemin de Longue Etude (éd. A. Tarnowski, Paris, Lettres Gothiques, 2000, v. 1725ss).

${ }^{11}$ Saint Ambroise, De Virginitate, XVIII, 115-116 évoque l'ascension vers Dieu : «Le mouvement porteur des ailes, ce n'est pas l'assemblage matériel des plumes, mais la succession continue des bonnes actions. Loin des honneurs de la vie militaire, loin des passions brûlantes de ce monde, que la cire fondue sous la chaleur brûlante du soleil, n'aille pas, comme le rapportent les mythes, abandonner les vols icariens, qui y perdraient leurs plumes ». Saint Augustin (Contra academicos, III, 2,3) fait aussi du vol d'Icare une image de l'âme. Exemples cités par M. Dancourt, op. cit., p. 29. On remarquera cependant que Dédale et Icare sont absents chez le grand passeur de mythes antiques qu'est Fulgence.

${ }_{12}$ On notera que saint Augustin fait référence à Dédale, non pour en faire un symbole de l'âme, mais comme exemple pour appuyer une réflexion sur la fable, qui ne peut être que fiction. Pour étayer cette idée, saint Augustin aurait pu utiliser n'importe quel mythe. Le choix de Dédale est cependant particulièrement pertinent dans la mesure où il associe les ailes artificielles et la fiction : « la fable du vol de Dédale ne peut être vraie que s'il est faux que Dédale ait volé » (Soliloquium, II, X, 18 : voir l'analyse de R. Dragonetti dans La musique et les lettres. Etudes de littérature médiévale, Genève, Droz, 1984, p. 403).
} 
Dans l'Ovide moralisé Dédale est en effet

Li bons fevres, li charpentiers,

Li trouverez de tous mestiers,

Li bons mestres, c'est Diex meïsmes,

Qui fist enfer et les abismes,

Si charpenta le firmament,

Et fist par son sens sagement

Le ciel, l'air et la terre et l'onde (1. VIII, v. 1767-1773).

Dédale est donc le charpentier divin, qui peut aussi être rapproché de Joseph (Matthieu 13$55)$.

Les deux ailes, loin de n'être que des artifices vains, reçoivent dans l'Ovide moralisé, malgré le peu d'efficacité que leur reconnaît le mythe, une lecture très valorisante car elles permettent d'atteindre le royaume des Cieux :

Par deus eles doivent voler

Cil qui la s'en vuelent aler.

L'amour de Dieu, c'est l'ele destre ;

L'amour du proisme est la senestre (v. 1819-1824).

A cette lecture chrétienne, s'oppose, dans une perspective qui témoigne de la plasticité de ce mythe au Moyen Âge, le discours de Raison dans le Roman de la Rose. Cette figure allégorique décrit les différentes sortes d'amour, dont l'amour des richesses. C'est à cette occasion que Jean de Meung introduit sa première fable mythologique, autour de Dédale ${ }^{13}$. La place stratégique de l'histoire d'Icare et Dédale dans le Roman de la Rose, qui fut l'un des plus grands succès du Moyen Âge, invite à la regarder de près ${ }^{14}$. Monnaie, représentée sous la forme d'une allégorie, tient les avares sous ses pieds, pour leur malheur, tandis que

Li vaillant homme l'assaillent

Et la chevauchent et porsaillent

Et tant as eperons la batent,

Qu'il s'en aaisent et esbatent,

Pour le cuer qu'il ont large et ample.

A Dedalus prennent essample,

Qui fist eles a Ycarus,
Quant par art et non pas par us

Tindrent par l'air voie commune.

Tout autel font cist a peccune,

Qui li font eles pour voler

Qu'ainz se lairoient affoler,

Qu'il n'en eüssent lous et pris. (v. 5217ss).

\footnotetext{
${ }^{13}$ Le Roman de la Rose, Paris, Lettres Gothiques, 1992, p. 303.

${ }^{14}$ Comme le note A. Strubel dans son édition, elle sera suivie de nombreux autres récits de ce type (environ quatre-vingts).
} 
Comme dans la parabole des talents, dont elle serait une version profane, l'histoire de Dédale et Icare fait l'éloge de celui qui fait circuler l'argent. L'analogie saute de la chevauchée au vol : n'en soyons pas surpris, dans la mesure où le vol d'Icare peut être rapproché de la navigation ${ }^{15}$ et où, plus que le medium c'est le déplacement qui compte dans la représentation.

L'histoire de Dédale et Icare est donc bien connue tout au long du Moyen Age : elle n'est pas bridée par une lecture consensuelle, elle n'est pas non plus topique. Présents dans Flamenca ${ }^{16}$, dans Athis et Prophilias, Berinus ou les Vøux du Paon, de l'allusion au résumé, d'autant plus riche que les divers aspects du mythe antique sont décomposés, isolés, Dédale et Icare étaient à la portée de l'auteur de Perceforest et ce d'autant plus qu'il aime construire des figures ambiguës et composites -il ne craint pas de mettre en scène un Zéphir des plus complexes, qui tient de Génius, d'Amour, du luiton épique et de l'ange gardien- et que les textes à partir desquels il travaille (le cycle du Paon et le Roman de la Rose en particulier ${ }^{17}$ ) font référence à Dédale et Icare. Pourquoi l'auteur de Perceforest ne s'est-il pas à son tour saisi de Dédale et Icare, pourquoi Bladud n'est-il pas devenu une figure matricielle dans Perceforest? Cette absence est aussi significative que les mentions que nous venons de relever et permet de mettre en évidence, à la fin du Moyen Âge, trois siècles après Hugues de Saint Victor, un traitement différencié des arts mécaniques.

II. L'artifice : charpente de marine et mise en scène théâtrale

Point d'avatar de Dédale ou Icare dans Perceforest alors même que l'auteur fait preuve d'un intérêt pour la mythologie très prérenaissant. Une première cause me semble être que la figure de Dédale (comme celle d'Icare) serait entrée en concurrence avec Zéphir, le luiton, qui est un «personnage» clé dans Perceforest. Comme j'ai tenté de le montrer dans mon livre Perceforest et Zéphir, cette fascinante créature a été inventée, entre autres, à partir du Génius ailé du Roman de la Rose qui doit beaucoup à Cupidon, du Zéphir d'Amour et Psyché et de l'ange gardien : on le voit souvent voler, pour transporter ses protégés, vers un lieu de sabbat, vers un lit accueillant, vers une mare fétide... Or, si l'on observe les représentations iconographiques des

\footnotetext{
${ }^{15}$ Voir mon article cit. note 1.

${ }^{16}$ Ed. Marion E. Porter, Princeton, 1962, p. 62.

${ }^{17}$ Pour ce qui est du rapport avec le cycle du Paon, voir Perceforest et Zéphir, op. cit., p. 33ss et J. Van der Meulen, «Simon de Lille et sa commande du Parfait du paon. Pour en finir avec le Roman de Perceforest », dans Patrons, Authors and Workshops: Books and Book Production in Paris Around 1400, éd. Godfried Croenen et Peter Ainsworth, Louvain, Paris et Dudley, Peeters, 2006, p. 223-238.
} 
anges, de Cupidon, de Génius, et d'Icare, on remarque qu'elles se confondent : toutes ces figures ont de grandes ailes d'oiseau, qui ne sont pas représentées de dos, ce qui évite l'épineux problème de l'attache de ces appendices. Les armatures de bois des ailes d'Icare et Dédale ne sont pas figurées : si le texte de l'Ovide moralisé faisant intervenir dans son texte le charpentier divin ne les néglige pas, les miniatures au contraire les escamotent, comme si le modèle surplombant de l'ange s'était imposé pour toutes les figures à forme humaine dotées d'ailes, qu'il s'agisse de Génius, de Dédale ou d'Amour. C'est ainsi que sont représentés sur le même modèle Génius dans le manuscrit BnF fr. 380 du Roman de la Rose (f. 125), Dédale dans le manuscrit BnF fr. 137 qui donne une traduction anonyme des Métamorphoses d'Ovide, contemporaine de Perceforest et originaire de la même zone ${ }^{18}$ (f. 106), Amour (par exemple dans le Roman de la Rose du XIVe siècle donné par le manuscrit BnF fr. 380 qui au folio $17 \mathrm{v}$ montre Amour et Amant, et au f. 8 Amour et Doux Regard). Sous le nom de Cupidon, on retrouve un personnage similaire dans le manuscrit BnF fr. 606 qui donne vers 1406 l'Epître Othea de Christine de Pizan (f. 23). Le modèle iconographique commun est celui de l'ange, tel que le représente par exemple le manuscrit BnF fr. 186 au f. 36 : l'ange gardien, tel qu'il est figuré par exemple dans le Livre des Anges de François Eximenez ${ }^{19}$, est le prototype à la fois d'Amour (Cupidon), Génius et Dédale $^{20}$. L'auteur de Perceforest, dont l'invention s'appuie certes sur les textes, mais aussi sur les images ${ }^{21}$, n'a peut-être pas pu développer le personnage de Dédale (ou celui d'Icare) parce qu'il manquait d'autonomie, de lisibilité, et que figuré comme Amour et Génius, il risquait de faire doublon avec Zéphir qui tient aussi de ces deux modèles.

D'autre part, l'auteur de Perceforest réserve les airs aux créatures surnaturelles : un homme comme Dédale n'y est pas à sa place. Mis à part le fugitif Bladud imposé par Geoffroy de Monmouth, l'auteur de Perceforest fait voler les mauvais esprits ( dans ce cas, il prend appui sur les représentations folkloriques de la Mesnie Hellequin nocturne ${ }^{22}$ ) et en particulier Zéphir, qu'il nous montre par exemple emportant Estonné dans les airs sur les lieux d'un sabbat. Les sorcières

\footnotetext{
${ }^{18}$ Ce manuscrit date des années 1450-1500 et a été réalisé dans les Flandres. La rubrique indique : comment Dedalus et son filz s'envolerent par l'air et comment le filz se noya en la mer par outrecuidance.

${ }^{19}$ Le site Mandragore sur lequel cette miniature est visible attribue par erreur l'œuvre à Pierre d'Artes, qui est en fait le commanditaire. L'auteur est frère François Eximinez (Dictionnaire bibliographique, historique et critique des livres rares, par R. Duclos, André-Charles Cailleau, Jacques-Charles Brunet, Paris, 1802, p. 424). Ce manuscrit est contemporain de Perceforest (vers 1475-1500), et réalisé à Bruges, il appartient à la même aire culturelle.

20 Toutes ces miniatures sont reproduites sur le site Mandragore de la Bibliothèque Nationale de France (http://mandragore.bnf.fr, consulté le 3 décembre 2011).

${ }^{21}$ Ainsi le modèle de la tour dans laquelle se trouvent une femme et une créature ailée, lui a servi à agglomérer l'Annonciation à Marie, Amour et Psyché, la naissance de Merlin et la reprise de celle-ci par Baudouin Butor.
} 
qui sont peintes à cette occasion ne volent pas sur des balais, mais sur des esprits diaboliques ${ }^{23}$. La Pucelle aux deux dragons, qui se déplace dans les airs, vraisemblablement inspirée par Médée (une figure positive en cette fin de Moyen Âge et particulièrement appréciée en milieu bourguignon), n’est pas une simple mortelle : elle est la petite-fille de Sébille, la demoiselle qui a enchanté Alexandre ${ }^{24}$. Quant à Galafur à qui on promet dans le livre V que Vénus (en fait une jeune parente de Morgue, qui se fait passer pour la déesse) viendra le rejoindre de son paradis en avolant, il est simplement victime d'une mise en scène ${ }^{25}$. Les humain(e)s ne volent pas de leurs propres ailes dans Perceforest : les airs sont réservés aux créatures surnaturelles, en particulier aux anges déchus, comme l'explique Zéphir à Estonné (1. II, t. 1, p. 73). On comprend dès lors pourquoi notre texte ne fait aucune allusion à l'exploration du ciel par Alexandre le Grand, alors qu'il réserve une place à l'exploration sous-marine ${ }^{26}$; on comprend peut-être aussi pourquoi la Pucelle aux deux dragons se nomme Alexandre ${ }^{27}$. Dédale et Icare, malgré les promesses de Bladud, n'avaient donc pas leur place dans Perceforest du fait de la concurrence exercée par d'autres figures, d'autres motifs, d'autres épisodes.

Cependant avec la variante introduite par David Aubert au texte de Geoffroy de Monmouth, s'ajoute une autre cause. Les artes mechanicae sont dévalorisées au Moyen Age. La fabrication des ailes par Dédale n'est pas seule à être mise à l'écart : d'autres activités comparables sont ostensiblement éliminées du récit par l'auteur. Sans qu'il soit possible de mener une étude exhaustive sur les arts mécaniques dans cette vaste fresque qu'est Perceforest, trois exemples

\footnotetext{
${ }^{22}$ Voir Perceforest et Zéphir, op. cit., p. 278ss.

${ }^{23}$ Voir mon article «Le sabbat de vieilles barbues dans Perceforest», dans Le Moyen Age, t. 99, 1993, p. 471-504.

${ }^{24}$ Voir mon article : «Sebille prophétesse et maternelle: du monde antique au monde arthurien dans Perceforest», dans La Sibylle, parole et représentation, éd. M. Bouquet et F. Morzadec, Presses Universitaires de Rennes, 2004, p. 211-225.

${ }^{25}$ Perceforest et Zéphir, op. cit., p. 156.

${ }^{26}$ Sur la tradition de ces deux vols, voir mon introduction dans Le roman d'Alexandre en prose du manuscrit Royal 15 E VI de la British Library, édition du texte, reproduction et analyse des miniatures, en collaboration avec Y. Otaka et H. Fukui, Tokyo, 2003 (trad. japonaise 2008), p. 37-39 et G. Millet, "L'ascension d'Alexandre », dans Syria, t. IV, 1923, p. 85-133. Sur l'exploitation de l'exploration sous-marine dans Perceforest, voir mon livre Fées, bestes et luitons, Paris, Presses de l'Université Paris Sorbonne, 2002, p. 299ss.

${ }^{27}$ On peut penser que l'auteur a donné à la demoiselle volant dans les airs le nom du Macédonien Alexandre, héros fondateur dans son roman : ainsi il ancrait son récit dans la tradition alexandrine (d'autant que la demoiselle descend du Macédonien). Cela permettait aussi de ne pas nier complètement l'exploration céleste d'Alexandre, connue de tous à l'époque et difficile à écarter, sans pour autant mettre en scène un simple humain -l'empereur- dans les airs.
} 
illustrent particulièrement bien la mise à l'écart des arts mécaniques : Alexandre et son tonneau, Sébille et son four à chaux ${ }^{28}$, Gloriande et son automate ${ }^{29}$. Nous ne développerons que le premier.

Reprenant la tradition alexandrine, l'auteur de Perceforest décrit l'artifice qui permit au Macédonien d'explorer les fonds marins : il s'estoit fait enclorre en ung tonnel de voirre si soubtillement que eaue ne pouoit entrer dedens et sy avoit air par buses, puis se fist avaler par dedens mer et mener a cordes par une nef aval la mer, car il vouloit sçavoir des merveilles qui sont dedens et comment les poissons se maintenoient (1. I, t. 1, §150).

Par rapport à la tradition fixée dans les versions en vers ${ }^{30}$, Perceforest ajoute un élément original qui témoigne d'un souci technique : les buses. Ce terme désigne un conduit pour l'écoulement des eaux : dans le Dictionnaire du Moyen Français (qui ignore l'occurrence de notre texte), deux uniques attestations sont relevées en ce sens. Dans le dictionnaire de Godefroy, est cité aussi un cas où buse désigne un trou dans un mur. Il s'agit donc d'un terme technique, qui d'ailleurs existe encore de nos jours. Cependant, malgré cette précision prometteuse, l'auteur de Perceforest focalise son bref résumé sur l'exploration et ne raconte pas la fabrication du bathyscaphe. En cela il est différent de son quasi contemporain (et peut-être rival), Jean Wauquelin, qui dans Les faicts et les conquestes d'Alexandre le Grand ${ }^{31}$, insiste sur la fabrication :

Ne demoura guaires aprés qu'il lui vint en voulenté de aller aussi bien cerquier le fons de la mer qu'il avoit cerquié l'ayr, pour veoir les merveilles qui sont dedens. Si fist tantost et incontinent venir a lui aucuns verriers, auquelz il proposa de faire ung tonnel de voirre si grant et si ample que bien se peut retourner dedens a sa voulenté, et avec ce si cler que bien peust veoir toutes choses parmy. Si le firent ainsi et ainsi. Ilz le firent tresbien loier de bonnes chaines de fer a ung gros annel par dessoubz pour y atachier une corde grosse de cavene. Quant il ot son tonnel appointié et ordonné a sa voulenté, il entra dedens et mist lui plusieurs lampes ardans et puis fist restouper le pertuis si bien que nulle goute d'ieaue n'y peust entrer. Aprés ce il se fist porter par vaisseaulx en le haute mer (\$218).

\footnotetext{
${ }^{28}$ Sebille est une fée bâtisseuse, comme Mélusine, mais on ne la voit pas à l'œuvre et ce qui est retenu de son activité c'est la fumée dégagée par les fours, qui enveloppent sa demeure d'une sorte de brouillard merveilleux. Sur les fours à chaux de Sebille, voir mon livre cit. Perceforest et Zéphir, p. 60.

${ }^{29}$ Sur Gloriande, qui invente des automates sans que sa pratique doit décrite, voir mon article "Gloriande dans Perceforest : entre effacement et fascination, une fée à la mode bourguignonne ? », à paraître dans les Actes du colloque de la société Rencesvals, «Les relations entre les hommes et les femmes dans la chanson de geste » (Lyon, 28 et 29 novembre 2011), sous la dir. de C. Füg-Pierreville.

${ }^{30}$ Voir par exemple The Medieval French Roman d'Alexandre, vol. II, Version of Alexandre de Paris, éd. E. C. Armstrong, D. L. Buffum, Princeton Paris, 1937, branche III, laisses 20-26, v. 389-577.

${ }^{31}$ Ed. S. Hériché, Genève, Droz, 2000, 1. II, chap. LX, §218, p. 456, et note p. 626.
} 
Chez Jean Wauquelion, l'artisan verrier est mentionné, les performances du produit sont précisées (taille du verre, transparence, étanchéité). La fin du Moyen Âge a développé un goût très marqué pour les verres : ce tonneau, quoique remontant à une longue tradition, prend en ce XVe siècle où toute l'Europe apprend des maîtres italiens un art du verre particulièrement raffiné, une résonance particulière. Le tonneau décrit par Wauquelin est la pièce de verre, irréalisable, dont rêve tout $\operatorname{artisan}^{32}$ et dont l'iconographie médiévale, à partir de 1450 s'empare, comme l'a montré M. Stanesco ${ }^{33}$, en représentant fréquemment de grosses boules de verre. L'auteur de Perceforest, s'il ajoute le détail technique de la buse, reste indifférent à la prouesse technique que constitue ce tonneau : son but est de motiver l'invention par Alexandre des tournois (inspirés par les combats des poissons chevaliers vus lors de l'exploration sous-marine) ${ }^{34}$. Que le tonneau soit tout à fait secondaire est confirmé par le deuxième épisode où il est question des poissons chevaliers : Bethidès les rencontre, sur un rivage marin ; il n'est plus besoin d'un tonneau puisque le chevaler se trouve sur la terre ferme (1. III, t. 2, p. 273ss).

Si la fabrication du verre et d'automates, la construction et l'architecture, qui ne sont toutes certes pas directement englobées dans l'énumération d'arts mécaniques d'Hugues de SaintVictor (qui, bridée par le chiffre sept, échoue à prendre en charge la diversité du réel) mais qui renvoient à des activités humaines comparables et intégrées à d'autres énumérations des arts mécaniques ${ }^{35}$, sont, comme les ailes de Dédale, tenues à l'écart dans Perceforest, ce n'est pas le cas, loin s'en faut, du théâtre ${ }^{36}$, un autre art mécanique selon Hugues de Saint Victor. Décrit en terme d'illusions et d'enchantements, d'engin et de faerie, le théâtre des fées et des diables est omniprésent dans Perceforest. Les mises en scène féeriques, qu'il s'agisse des banquets et de leurs entremets, des entrées royales, des fantasmagories établies par les fées ou par Aroés

\footnotetext{
${ }^{32}$ Les miniatures qui illustrent la fabrication du verre rendent bien compte de la petite taille des objets à l'époque de Perceforest: voir par exemple le Livre des simples médecines, BnF fr.12319, f. 316 (France du Nord, vers 1475). Des illustrations tout à fait similaires se retrouvent régulièrement pour cet ouvrage (Livre des simples médecines, BnF fr. 19081, f. 202, Flandres, milieu $15^{\mathrm{e}}$ s) ou pour d'autres œuvres qui traitent le même sujet, comme le Tractatus de herbis (BnF Latin 6822, f. 132, manuscrit du XVe s.).

33 «Une merveille bien énigmatique : le chevalier dans un tonneau de verre», dans Le monde et l'autre monde, textes réunis par C. Ferlampin-Acher et D. Hüe, Orléans, Paradigme, 2002, p. 359-368. M. Stanesco s'intéresse à la multiplication vers 1450 dans l'iconographie des cages et boules de verre, déjà notée par J. Baltrusaitis (Le Moyen Âge fantastique. Antiquités et exotismes dans l'art gothique, 1955, rééd. Paris, 1981, p. 194-202).

${ }^{34}$ Sur cette invention, voir mon livre Perceforest et Zéphir, op. cit., p. $97 \mathrm{ss}$.

${ }^{35}$ Voir en particulier Raymond Lulle, dans son Llibre de la Contemplacio qui intègre la forge (ferreria) et la construction (fusteria), qui correspondent aux activités de Gloriande et ses automates mécaniques et Sebille la bâtisseuse. Voir A. Llinares, « Le travail manuel et les arts mécaniques chez Raymond Lulle », dans Raymond Lulle et le pays d'Oc, Cahiers de Fanjeaux, t. 22, 1987, p. 169-189.
} 
l'enchanteur ${ }^{37}$, sont nombreuses, où merveilleux et théâtralité vont de pair, comme souvent dans les récits médiévaux dans lesquels la merveille est avant tout regard problématique, vision incertaine et déformée, troublée et perturbée. Les dispositifs concrets appuyant la magie sont rarement décrits, sauf dans le cas de l'enchanteur Aroés, diabolique, qui se pose en rival de Dieu et finit emporté par des démons ${ }^{38}$ ou dans celui du Chastel Desvoyé. Dans ces deux cas, le dispositif est optique : possédant un anneau qui lui permet d'échapper aux enchantements et qui fait de lui l'égal du narrateur omniscient, Gadifer ne succombe pas à l'illusion établie par Aroés et en voit, au contraire, les causes optiques et mécaniques, des fioles toutes plaines d'eaues sur lesquelles se reflète la lumière, un cercle de fer de merveilleuse grandeur que l'enchanteur peut manipuler du doigt pour faire tourner la salle, des ampoulles de voirre où joue la flamme des torsis. L'ambiguïté merveilleuse est maintenue, car ces explications optiques n'excluent pas la magie noire : les ampoules sont toutes plaines de merveilleuses eaues faittes par art mauvais (1. III, t. 1, p. 81ss). Un dispositif comparable permet au Chastel Desvoyé de devenir invisible ${ }^{39}$. Ces manifestations, toutes plus ou moins inquiétantes même lorsqu'elles sont mises en scène par la Reine Fée, renvoient les arts mécaniques définitivement du côté de l'inhumain, du mystère, de la rivalité entre l'homme et Dieu. Elles ont pour but de tromper : Aroés, grâce au dispositif qu'il a mis en place, fait croire à ses sujets qu'il est un dieu et il prétend leur montrer leurs proches en Enfer et au Paradis ${ }^{40}$; les nièces de Morgane ont décidé de construire un engin en se faisant passer pour Vénus afin de sélectionner le meilleur chevalier pour en avoir un enfant : elles trompent les chevaliers de passage en prétendant que Vénus viendra de son paradis, comme on montrerait au théâtre un ange surgissant du ciel grâce aux secrez (qui sont les effets spéciaux de l'époque). Ces machines, ces engins, ne sont que des machinations, qui abusent les sens et l'entendement. Elles sont aussi le reflet d'une société de spectacle, celle de la Bourgogne de Philippe le Bon, placée sous le signe du jeu, de l'artifice, de la fête, entre les automates et les jeux d'eau du Parc

\footnotetext{
${ }^{36}$ Le théâtre est exclu des arts mécaniques par Llull. Hugues de Saint-Victor relève au contraire parmi ces arts la theatrica scientia, tout comme Vincent de Beauvais.

${ }^{37}$ Sur ces épisodes et leur relation au théâtre, voir mon article «Perceforest ou quand les diables font du théâtre dans un roman », dans Le Jeu et l'Accessoire. Mélanges en l'honneur du professeur Michel Rousse, textes réunis par M. Bouhaïk-Gironès, D. Hüe et J. Koopmans, Paris, Garnier, 2011, p. 351-368.

${ }^{38}$ Voir D. Delcourt, «Magie, fiction et phantasme dans le Roman de Perceforest: pour une poétique de l'illusion au Moyen Âge », dans Romanic Review, t. 85, 1994, p. 167-178 et J. Taylor, « Aroès the enchanter: an episode of the Roman de Perceforest and its sources », dans Medium Aevum, t. 47, 1978, p. 30-39.

${ }^{39}$ Voir mon article «Les déceptions dans Perceforest: du fantosme au fantasme», dans Félonie, trahison, reniements au Moyen Age. Actes du troisième colloque international de Montpellier. Université Paul Valéry (24-26 novembre 1995), Les Cahiers du C.R.I.S.I.M.A., 3, 1997, p. 423ss.

${ }^{40}$ Voir mon livre Fées, bestes et luitons, op. cit., « Aroès dans Perceforest : l'opticien du diable », p. 184ss.
} 
d'Hesdin et les entremets des Vœux du Faisan. Les arts mécaniques, quoique inscrits dans l'orbite des fastes curiaux auxquels ils sont devenus nécessaires, sont considérés avec la fascination et l'inquiétude d'un lettré, qui ne veut pas savoir comment l'on fabrique, pour mieux s'ébahir, pour entretenir la superbe aristocratique loin de la matière... A moins que l'exclusion des savoirs faire techniques ne permette de mettre en valeur l'unique artifice digne de rivaliser avec Dieu : la fiction, la fable.

Icare et Dédale, tirés par David Aubert du côté de la navigation et des arts mécaniques, n'avait donc aucune chance de devenir une figure matricielle dans ce vaste roman qu'est Perceforest. Malgré le succès médiéval que connurent Icare et Dédale, d'une part parce qu'ils exprimaient un rêve profondément ancré en l'homme, d'autre part parce que le mythe, conservant sa plasticité, n'avait pas été enfermé dans une lecture topique et univoque, Dédale-Bladud entrant en concurrence avec Zéphir et, n’étant appuyé par aucune représentation spécifique sur le plan iconographique, resta confiné aux marges du récit, dans la traduction de l'Historia regum Britaniae de Geoffroy de Monmouth. Plus profondément, tiré du côté des arts mécaniques, le vol d'Icare ne pouvait qu'être marginal : Perceforest tient à l'écart les arts mécaniques dans leur ensemble, qu'il s'agisse de la construction ou de la forge. Seul le théâtre échappe à cette réticence, mais quoique fort présent, il n'en est pas pour autant valorisé en tant que savoir-faire «mécanique », puisqu'il est systématiquement tiré du côté de la magie, de l'enchantement et de l'illusion optique. Dans cette société curiale de la deuxième moitié du XVe siècle, consommatrice de spectacles et de produits manufacturés, Perceforest semble donc marqué par le préjugé, à la fois nobiliaire et clérical, d'arts mécaniques méprisables, tandis que l'artiste tend à se dégager de l'artisan. Dédale l'ingénieur, pas plus que l'artisan, forgeron ou charpentier de marine, ne pouvait pas devenir chevalier et intégrer un roman; un opticien, maître verrier fabriquant des fioles comme Aroès, ne pouvait être clerc. L'artifice, qu'il soit artisanal ou artistique, est dévalorisé : parce qu'il est du côté de la matière et du corps, et non de l'esprit, parce qu'il rivalise avec Dieu et fait courir le risque de l'illusion diabolique. L'artisanat est exclu du récit, l'illusion théâtrale au contraire l'envahit : le goût de l'aristocratie bourguignonne, avide de spectacles, et l'intérêt du poète pour la fiction se rejoignent. 